\title{
A contribuição da comunicação alternativa PECS - (método por troca de figuras) na comunicação funcional de crianças autistas
}

\author{
The contribution of alternative communication PECS - (method for exchanging figures) in the
} functional communication of autistic children

La contribución de la comunicación alternativa PECS - (método de intercambio de figuras) en la comunicación funcional de los niños autistas

Recebido: 08/01/2021 | Revisado: 11/01/2021 | Aceito: 14/01/2021 | Publicado: 17/01/2021

Francisca Wérica Teixeira Luz

ORCID: https://orcid.org/0000-0002-3169-8558 Faculdade de Ensino Superior do Piauí, Brasil E-mail: wericaluz2020@gmail.com

Aracy Teresa Castelo Branco

ORCID: https://orcid.org/0000-0002-5277-1582 Faculdade de Ensino Superior do Piauí, Brasil E-mail: aracycoelho@yahoo.com

\begin{abstract}
Resumo
$\mathrm{O}$ presente trabalho tem como objetivo geral analisar o aumento das interações funcionais da criança com a aplicabilidade da comunicação alternativa - PECS (método com trocas de figuras) com crianças autistas para a estimulação da comunicação verbal e funcional; e como objetivos específicos: elencar a eficácia e os desafios encontrados com a prática da comunicação alternativa- PECS com crianças autistas; analisar o número de pedidos funcionais de interações verbais/funcionais; observar os resultados da pratica da comunicação alternativa PECS, com crianças autistas. Para atingir tais objetivos, buscou embasamento teórico em autores como Vygotsky (2001) Walter e Nunes (2008) Corrêa Netto (2013), entre outros. A abordagem escolhida para fundamentar o estudo e atingir os objetivos, foi a revisão da produção bibliográfica de literatura. O estudo qualitativo foi elaborado a partir de dados presentes em artigos científicos selecionados e publicados no banco de dados de sites como: Bireme, Scielo e Google Acadêmico e em revistas cientificas, utilizando artigos de 2015 a 2020.Os estudos analisados nos permitiram sistematizar aportes teóricos e contextuais sobre a dimensão da comunicação alternativa PECS, como elemento primordial no desenvolvimento da linguagem, interação dos sujeitos e desenvolvimento humano. Os resultados mostraram maior interação comunicativa do aluno na fase de intervenção, além de generalizar o uso do PECS-Adaptado na sala de aula regular. Concluiu-se que a comunicação é um dos fatores fundamentais para que a inclusão escolar de um aluno com TEA ocorra de forma mais efetiva.
\end{abstract}

Palavras-chave: Autismo; Comunicação alternativa; PECS; Troca de figuras.

\begin{abstract}
The present work has as general objective to analyze the increase of the functional interactions of the child with the applicability of alternative communication - PECS (method with exchanges of figures) with autistic children for the stimulation of verbal and functional communication; and as specific objectives: list the effectiveness and challenges encountered with the practice of alternative communication - PECS with autistic children; analyze the number of functional requests for verbal / functional interactions; observe the results of the practice of alternative PECS communication with autistic children. To achieve these goals, he sought theoretical support from authors such as Vygotsky (2001) Walter and Nunes (2008) Corrêa Netto (2013), among others. The approach chosen to support the study and achieve the objectives, was the review of the bibliographic production of literature. The qualitative study was elaborated from data present in selected scientific articles and published in the database of sites such as: Bireme, Scielo and Google Scholar and in scientific journals, using articles from 2015 to 2020. The analyzed studies allowed us to systematize theoretical contributions and contextual about the dimension of alternative communication PECS, as a fundamental element in language development, subject interaction and human development. The results showed a greater communicative interaction of the student in the intervention phase, in addition to generalizing the use of PECSAdapted in the regular classroom. It was concluded that communication is one of the fundamental factors for the school inclusion of a student with ASD to occur more effectively.
\end{abstract}

Keywords: Autism; Alternative communication; PECS; Exchange of figures. 


\section{Resumen}

El presente trabajo tiene como objetivo general analizar el incremento de las interacciones funcionales del niño con la aplicabilidad de la comunicación alternativa - PECS (método con intercambios de figuras) con niños autistas para la estimulación de la comunicación verbal y funcional; y como objetivos específicos: enumerar la efectividad y los desafíos encontrados con la práctica de la comunicación alternativa - PECS con niños autistas; analizar el número de solicitudes funcionales para interacciones verbales / funcionales; observar los resultados de la práctica de la comunicación PECS alternativa con niños autistas. Para lograr estos objetivos, buscó el apoyo teórico de autores como Vygotsky (2001) Walter y Nunes (2008) Corrêa Netto (2013), entre otros. El enfoque elegido para apoyar el estudio y lograr los objetivos, fue la revisión de la producción bibliográfica de la literatura. El estudio cualitativo se elaboró a partir de datos presentes en artículos científicos seleccionados y publicados en la base de datos de sitios como: Bireme, Scielo y Google Scholar y en revistas científicas, utilizando artículos de 2015 a 2020. Los estudios analizados permitieron sistematizar aportes teóricos y contextual sobre la dimensión de la comunicación alternativa PECS, como elemento fundamental en el desarrollo del lenguaje, la interacción del sujeto y el desarrollo humano. Los resultados mostraron una mayor interacción comunicativa del alumno en la fase de intervención, además de generalizar el uso de PECS-Adaptado en el aula regular. Se concluyó que la comunicación es uno de los factores fundamentales para que la inclusión escolar de un estudiante con TEA ocurra de manera más efectiva.

Palabras clave: Autismo; Comunicación alternativa; PECS; Intercambio de figuras.

\section{Introdução}

De acordo com a American Psychiatric Association (2013), os critérios diagnósticos para transtornos do espectro do Autismo (TEA) abrangem um continuo comprometimento das capacidades de interação social, comunicação e de comportamentos, interesses e atividades repetitivas e restritas, a falha na linguagem e comunicação, tem um papel central na caracterização do TEA, uma vez que o déficit na comunicação e no desenvolvimento da linguagem está relacionado a falta de reciprocidade social e incapacidade de desenvolver e manter seus relacionamentos apropriados ao seu nível de desenvolvimento (American Psychiatric Association, 2013).

Por seus prejuízos na comunicação expressiva e receptiva, crianças com autismo são consideradas bons candidatos ao uso da Comunicação suplementar e/ou alternativa (CSA), com intervenções educacionais que visem o ensino de comunicação que são evidentemente bastante relevantes para que a criança adquira controle e participação em seu mundo social. Desta forma, a prancha de comunicação é um dos diversos recursos de baixa tecnologia disponíveis para a implementação de um sistema de comunicação alternativa (CA). Trata se de um tipo de recurso que pode ser confeccionado com materiais de custo acessível, como fotos, figuras de jornais e revistas, desenhos manuais etc.

O Picture Exchang Communication System (PECS), desenvolvido por Bondy e Frost (1994) é um método de intervenção em comunicação suplementar e/ou alternativa (CSA) baseado em princípios comportamentais básicos (modelagem, reforço diferencial e transferência de controle de estímulos, por exemplo) no qual a criança aprende a requisitar objetos ou atividades de interesse por meio da troca de figuras pelos itens (potenciais reforçadores).

O protocolo de ensino é baseado no comportamento verbal (Skinne, 1958) de tal forma que os operantes verbais funcionais são sistematicamente ensinados, usando estratégias de reforço que levaram a uma comunicação independente. O acompanhante não poderá dar dicas, apenas indagar a criança, construindo assim um início imediato do processo e evitando a dependência de dicas (Pecs Brasil, 2019).

Diante disso, iremos analisar as contribuições da utilização da Comunicação Alternativa - PECS - método por troca de figuras, para estimulação da comunicação funcional, interação social e autonomia das crianças com autismo. Dito isto, tem-se como objetivo geral deste trabalho, analisar o aumento das interações funcionais da criança com a aplicabilidade da comunicação alternativa - PECS (método com trocas de figuras) com crianças autistas para a estimulação da comunicação verbal e funcional; e como objetivos específicos: elencar a eficácia e os desafios encontrados com a prática da comunicação alternativa- PECS com crianças autistas; analisar o número de pedidos funcionais de interações verbais/funcionais; observar os resultados da pratica da comunicação alternativa PECS, com crianças autistas. Sendo assim, o trabalho foi norteado pelo seguinte questionamento a 
respeito do tema proposto: Quais as contribuições oferecidas pela Comunicação Alternativa - PECS - no que diz respeito à utilização de troca de figuras para estimular a comunicação funcional, interação social e autonomia de crianças autistas?

Diante destas expectativas, buscamos mostrar o efeito benéfico desse tipo de método no que concerne ao melhor desenvolvimento intelectual e sociocultural da criança autista e, porque não dizer, um artifício que vem chamando a atenção da sociedade em geral durante o processo de aplicabilidade da comunicação alternativa e que traz autonomia do autista ao se dispor a esta prática.

\section{Referencial teórico}

\subsection{PECS: Uma alternativa de intervenção na área de linguagem de crianças com autismo}

Sabe-se que a comunicação humana é de suma importância para que ocorra o processo de interação social. É por meio dessa comunicação que os indivíduos conseguem trocar experiências, conseguem manifestar seus desejos, anseios, vontades, gerando assim, aprendizagens intrínsecas que os permite participar mais ativamente da sociedade ao qual estão inseridos.

Dessa forma, pode-se dizer segundo Vygotsky (2001) que a comunicação assume duas funções principais, comunicar e compreender o que está sendo expresso e ainda assim, representar o pensamento. Logo, o processo de apropriação da linguagem, é de extrema importância para o desenvolvimento humano, pois é através da interação social, do convívio com o meio, que se adquire a linguagem e onde o cognitivo vai evoluindo conforme vá havendo experiências, se apropriando do conhecimento.

Sabendo disso, procura-se através deste trabalho, mostrar a importância de se trabalhar métodos que facilitem a comunicação de sujeitos que possuem TEA (Transtorno do Espectro do Autismo). Assim, indivíduos que apresentam o Transtorno do Espectro do Autismo possuem dificuldades bastante significativas na linguagem e, consequentemente na comunicação. Dessa forma, percebemos que as complexidades nas intenções comunicativas também podem contribuir para dificultar o processo de interação e relação social. Para Walter e Nunes (2008) os distúrbios na comunicação começam a ser percebidos associadamente com o desenvolvimento da criança, ou seja, desde os seus primeiros anos de vida. Tais problemas de linguagem podem trazer danos significativos ao seu desenvolvimento global.

Assim sendo, apresentamos neste trabalho o PECS - Picture Exchange Communication System (PECS) é um sistema muito utilizado para a comunicação de autistas, que ressalta a relação interpessoal, em que ocorre um ato comunicativo entre o indivíduo com dificuldade de fala e um adulto, por meio de troca de figuras, e estimulação com apoio visual, em uma sequência lógica permitindo ao autista expressar seus desejos e sentimentos. (Viera, 2019).

Em resumo, o PECS foi descrito originalmente em sete fases de treinamento e cada uma delas possui seu objetivo específico. Atualmente o programa se apresenta em seis fases de treinamento. Assim que o objetivo de cada fase é atingido, a criança avança para a fase seguinte do sistema. Vale ressaltar que a criança progride nas fases seguintes mediante alguns requisitos determinados pelo próprio manual de instruções do PECS.

Por certo, sofreu algumas modificações, entre elas foi que, ao contrário do PECS original, o PECS - Adaptado é disposto em cinco fases de comunicação, fundamentada no CFN, sendo que cada fase também possui seu objetivo específico, mediante as complexidades do desenvolvimento de linguagem infantil. Visto que as diferentes especificidades encontradas nas características de pessoas com TEA, principalmente no que se refere à comunicação e à interação social desta população e entendendo que cada uma delas é motivo para muitos estudos e discussões, percebe-se o quanto é importante desenvolver estratégias que facilitem o desenvolvimento desses sujeitos.

Nesse sentido, Corrêa Netto (2013) defende a importância de se difundir as singularidades no desenvolvimento dos alunos com TEA, além de oferecer estratégias e recursos no intuito de favorecer a prática de ações pedagógicas consistentes, beneficiando a aprendizagem e potencializando suas habilidades, para que a inclusão desse individuo realmente se efetive dentro 
da sociedade. Contudo, o incentivo à comunicação é essencial para o desenvolvimento social e cognitivo de indivíduos com TEA que venham apresentar disfunções na fala, contribuindo para facilitar no processo da linguagem e interação. A criança precisa ser observada de acordo com suas manifestações e necessidades e de acordo com sua idade, seu cognitivo em cada fase.

Logo, atualmente, existem diversos programas de comunicação alternativa para crianças com autismo e/ou dificuldades de fala, embora não haja, até o momento, um guia para clínicos que determine qual estratégia ou sistema de comunicação seja o mais efetivo para indivíduos com autismo e/ou outras dificuldades de fala (Flippin; Resca; Watson, 2010). Como ainda não se sabe qual sistema de comunicação alternativa e o mais efetivo, pesquisas, como as revisões bibliográficas, mostram sua relevância na organização dos dados existentes sobre a implementação e a intervenção dos diferentes sistemas de comunicação.

Desta forma, a rápida aprendizagem das habilidades envolvidas no PECS ocorre devido ao contexto estruturado e concreto desse treino que facilita a compreensão da comunicação funcional pelos indivíduos com autismo. Muitos estudos como o de Habani et al. (2002), mostram que quanto mais concreto, ou seja, com mais características físicas, estruturada e específica for o tipo de dica, melhor e mais rápido e o aprendizado de habilidades verbais de sujeitos autistas, se comparado com as dicas auditivas.

O presente estudo teve como propósito de analisar a eficácia do método PECS, com crianças autistas, para estimulação da comunicação funcional, através de uma revisão bibliográfica.

\section{Metodologia}

Existem vários tipos de pesquisa, mas a abordagem escolhida para fundamentar este estudo e atingir os objetivos, optouse pelo método da revisão bibliográfica integrativa de literatura. A abordagem é do tipo qualitativa. Para entender a natureza desse tipo de pesquisa nos baseamos em Minayo (2007, p. 21) quando diz que [...] "a pesquisa qualitativa responde a questões muito particulares. Ela se preocupa com um nível de realidade que não pode ser quantificado.

O presente estudo qualitativo foi elaborado a partir de revisão narrativa, baseado em dados presentes em artigos científicos selecionados e publicados no banco de dados de sites como: bireme, scielo e google acadêmico e em revistas cientificas, utilizando artigos de 2015 a 2020. Isso se deve ao fato de dispor de publicações relevantes que discutissem o assunto que é essencial para a execução da pesquisa. Para tanto, a busca nos bancos de dados foi realizada utilizando palavras ou expressões específicas como, pecs, autismo e comunicação alternativa. Com isso, a primeira ação foi descartar aqueles textos que não se incluíam na área da pesquisa em estudo.

Adotaram-se os seguintes critérios de inclusão para a seleção dos artigos analisados: crianças de até 10 anos de idade e artigos originais escritos em português com disponibilidade de texto completo disponível na íntegra em suporte eletrônico, publicado em periódicos nacionais, livros, teses, anais de congressos ou conferências e publicado nos últimos cinco anos. A predileção por esse recorte temporal comprova-se pelo fato de esta revisão basear-se em uma literatura atualizada, considerando que as informações veiculadas em mídias informatizadas circulam atualmente com maior rapidez. Já os critérios de exclusão foram: capítulos de livros, capítulos de teses, artigos publicados em periódicos não editados no Brasil, relatórios técnicos e científicos e documentos ministeriais, bem como crianças em idade menor que 10 anos de idade. Assim, envolveu atividades de busca, identificação, fichamento de estudos, mapeamento e análise.

$\mathrm{O}$ trabalho se enquadra quanto aos objetivos como sendo uma pesquisa do tipo descritiva, sendo a mais tradicional das pesquisas, está interessada em descobrir o que acontece, ela descreve as características de uma determinada população ou determinados fenômenos e os interpreta, não busca interferir, nem modificar a realidade estudada (Rudio, 2001).

Ademais, a busca foi executada nos meses de agosto a outubro de 2020, empregando a revisão da produção bibliográfica da literatura. Essa pesquisa reúne, analisa e discute informações a partir dos mais relevantes estudos originais de revisão que 
demonstram resultados referentes à comunicação alternativa PECS com crianças autistas. É feita a partir do levantamento de referências teóricas já analisadas, e publicadas por meios escritos e eletrônicos, como livros, artigos científicos, páginas de web sites.

Os dados foram organizados em categorias de acordo com suas similaridades, as categorias são concentradas para se instituir e classificar os elementos, as ideias ou expressão que seja capaz de abranger uma totalidade e chegar a uma resposta que seja ela negativa ou positiva. Essa etapa busca uma leitura que seja efetiva e meticulosa de cada artigo selecionado para analisar o ajustamento ao tema, sua relevância, autenticidade e profundidade e por fim, foram fichados e organizados com intuito de responder o objetivo pelo estudo.

\section{Resultados}

Sabe-se que uma das principais características do autismo é no transtorno da comunicação, as disfunções qualitativas na interação social, além de comportamento repetitivo ou estereotipado. Logo, o método apresentado no trabalho, busca contribuir com a melhoria e uma melhor qualidade de vida de pessoas com TEA, aumentando sua autonomia e a inclusão social destas.

Consoante, os estudos analisados nos permitiram sistematizar aportes teóricos e contextuais sobre a dimensão da comunicação alternativa PECS, como elemento primordial no desenvolvimento da linguagem, interação dos sujeitos e desenvolvimento humano.

A análise dos aportes teóricos nos permitiu sistematizar pressupostos para uma abordagem que estamos denominando de psicossocial e nos fez compreender o quanto o uso do PECS-adaptado contribui para a melhoria de diversos aspectos da vida da criança autista. Mais ainda, ao se analisar o conjunto da amostra verifica-se que há prevalência de poucos estudos científicos que abordam sobre a utilização do método de comunicação alternativa PECS com portadores do transtorno do espectro do autismo. Essa área se encontra em processo de desenvolvimento e investigação, o que denota a necessidade de haver mais pesquisas neste âmbito que possam contribuir para a intervenção e o trato de portadores com autismo.

Os resultados serão apresentados de acordo com o objetivo geral deste estudo que é analisar a produção bibliográfica de trabalhos científicos relacionados à aplicação do método PECS em indivíduos com transtorno do espectro do autismo.

Abaixo são demonstrados os objetivos de alguns autores, bem como suas metodologias e os resultados que obtiveram com suas respectivas pesquisas.

Quadro 1. Contribuições de autores sobre a aplicação do método PECS em indivíduos com TEA.

\begin{tabular}{|c|c|c|c|}
\hline $\begin{array}{l}\text { Autor/ano da } \\
\text { publicação }\end{array}$ & Objetivos & Metodologias & Resultados \\
\hline $\begin{array}{l}\text { Oliveira et al } \\
\quad(2015)\end{array}$ & $\begin{array}{l}\text { O objetivo do estudo foi o } \\
\text { de analisar a produção } \\
\text { bibliográfica de trabalhos } \\
\text { científicos relacionados à } \\
\text { aplicação do método PECS } \\
\text { em indivíduos com TEA. }\end{array}$ & $\begin{array}{l}\text { Optou-se pelo método da revisão da } \\
\text { produção bibliográfica da literatura. } \\
\text { O levantamento bibliográfico foi } \\
\text { realizado pela Internet nas seguintes } \\
\text { bases de dados e banco de teses: pela } \\
\text { BIREME, CEFAC, LILACS, } \\
\text { CAPES. }\end{array}$ & $\begin{array}{l}\text { Os resultados das pesquisas revisadas } \\
\text { neste estudo mostram claramente que o } \\
\text { PECS parece se mostrar um método de } \\
\text { ensino de linguagem eficaz. } \\
\text { A análise dos artigos obteve coerência nos } \\
\text { resultados da utilização do método PECS } \\
\text { com indivíduos autistas, sendo possível } \\
\text { perceber: há melhora nos aspectos } \\
\text { práticos da linguagem oral destes } \\
\text { indivíduos; desenvolvimento da interação } \\
\text { social; aumento na frequência de } \\
\text { verbalizações; ampliação no discurso } \\
\text { verbal e avanço no comportamento sócio } \\
\text { comunicativo. }\end{array}$ \\
\hline
\end{tabular}




\begin{tabular}{|c|c|c|c|}
\hline $\begin{array}{l}\text { Moreschi e } \\
\text { Almeida } \\
\text { (2012) }\end{array}$ & $\begin{array}{l}\text { Teve por objetivo planejar, } \\
\text { aplicar e avaliar os efeitos } \\
\text { de um programa de } \\
\text { intervenção sobre } \\
\text { Comunicação Alternativa } \\
\text { para o desenvolvimento de } \\
\text { habilidades comunicativas } \\
\text { verbais de uma adolescente } \\
\text { com deficiência intelectual. }\end{array}$ & $\begin{array}{l}\text { Foi utilizado o Delineamento de } \\
\text { Sujeito Único, AB, por ser uma } \\
\text { forma simples de se estabelecer } \\
\text { relação entre a variável dependente } \\
\text { e a variável independente em } \\
\text { fenômenos pouco explorados, sendo } \\
\text { utilizados em projetos de } \\
\text { intervenção, como em investigações } \\
\text { clínicas. Participou da pesquisa uma } \\
\text { adolescente (J) de } 14 \text { anos, } \\
\text { diagnosticada segundo avaliação } \\
\text { Psicológica com deficiência } \\
\text { intelectual e distúrbio de } \\
\text { comunicação }\end{array}$ & $\begin{array}{l}\text { O objetivo do presente estudo foi } \\
\text { alcançado, na medida em que a utilização } \\
\text { do Sistema de Comunicação Alternativa } \\
\text { possibilitou à participante ampliar suas } \\
\text { habilidades comunicativas. Ao utilizar tal } \\
\text { sistema, ela foi capaz de solicitar objetos } \\
\text { que desejava e assim, estabelecer uma } \\
\text { comunicação eficaz com outras pessoas, } \\
\text { fazendo do recurso de comunicação } \\
\text { alternativa um apoio à sua comunicação } \\
\text { oral. }\end{array}$ \\
\hline $\begin{array}{l}\text { Nunes e } \\
\text { Santos } \\
(2015)\end{array}$ & $\begin{array}{l}\text { O objetivo do presente } \\
\text { estudo consistiu em avaliar } \\
\text { a eficácia de uma adaptação } \\
\text { do protocolo Pecs e das } \\
\text { estratégias do AMI para o } \\
\text { desenvolvimento r da } \\
\text { comunicação de uma } \\
\text { criança autista de cinco } \\
\text { anos, tendo-se uma } \\
\text { professora como agente de } \\
\text { intervenção. }\end{array}$ & $\begin{array}{l}\text { No estudo foi utilizado um diário de } \\
\text { campo, onde foram registradas as } \\
\text { impressões e anotações da } \\
\text { pesquisadora (segunda autora). } \\
\text { A presente pesquisa foi conduzida } \\
\text { com Pedro, um aluno com } \\
\text { diagnóstico de autismo, e Neusa, sua } \\
\text { professora de apoio pedagógico. } \\
\text { Pedro tinha cinco anos e estava } \\
\text { matriculado no nível IV da } \\
\text { Educação Infantil }\end{array}$ & $\begin{array}{l}\text { Os dados da presente pesquisa sugerem } \\
\text { que a mera exposição desses indivíduos } \\
\text { aos recursos da CAA pode ser pouco } \\
\text { eficaz para o desenvolvimento de novas } \\
\text { formas de expressão. Apesar da presença } \\
\text { dos pictogramas em sala de aula, foram } \\
\text { escassas as ocasiões em que Pedro } \\
\text { utilizou os cartões para comunicar seus } \\
\text { desejos durante a fase de linha de base. A } \\
\text { professora, por sua vez, antes da } \\
\text { intervenção, empregava os pictogramas } \\
\text { essencialmente para ilustrar as rotinas em } \\
\text { sala de aula. Eram poucas as } \\
\text { oportunidades ofertadas ao aluno para } \\
\text { usar os pictogramas como forma de } \\
\text { comunicação expressiva. Nesse contexto, } \\
\text { a pesquisadora propôs a Neusa o } \\
\text { programa de capacitação no uso da } \\
\text { Comunicação Alternativa por meio do } \\
\text { Pecs e das estratégias naturalísticas } \\
\text { derivadas do modelo AMI. }\end{array}$ \\
\hline $\begin{array}{c}\text { Cabral et al } \\
\text { (2019) }\end{array}$ & $\begin{array}{l}\text { Teve como objetivo, relatar } \\
\text { a ação educativa executada } \\
\text { com intuito de apresentar o } \\
\text { PECS aos profissionais do } \\
\text { local como uma proposta } \\
\text { de ensino e melhoria na } \\
\text { comunicação dos } \\
\text { pacientes, assim refletindo } \\
\text { na qualidade de vida em } \\
\text { comunidade e para os pais } \\
\text { como facilitador do veículo } \\
\text { de comunicação verbal. }\end{array}$ & $\begin{array}{l}\text { Trata-se de um relato de experiência } \\
\text { de cunho qualitativo, elencado no } \\
\text { método de orientação e da } \\
\text { interpretação do programa, } \\
\text { relacionado a acessibilidade de } \\
\text { informações sobre a inserção desse } \\
\text { meio de comunicação na rotina da } \\
\text { criança. Estiveram presentes } 11 \\
\text { pais, } 4 \text { profissionais e } 2 \text { crianças } \\
\text { com TEA. A metodologia foi } \\
\text { aplicada em uma criança com TEA } \\
\text { que compareceu acompanhada da } \\
\text { mãe. }\end{array}$ & $\begin{array}{l}\text { A ação educativa possibilitou um impacto } \\
\text { positivo no público alvo, que por meio de } \\
\text { uma medida lúdica e educativa, o } \\
\text { aprendizado da criança é possível, visto } \\
\text { que os mesmos demonstraram interesse e } \\
\text { se comprometeram a alcançar o objetivo } \\
\text { trazido pela pasta. Esse modelo de } \\
\text { comunicação os ensina como deve ser } \\
\text { estimulada a verbalização da vontade da } \\
\text { criança, passando por todas as seis fases } \\
\text { necessárias, que por fim, torna-a } \\
\text { independente da pasta para poder se } \\
\text { comunicar. }\end{array}$ \\
\hline
\end{tabular}

Fonte: Autores (2020).

\section{Discussão}

De acordo com os estudos podemos perceber que os resultados dos textos selecionados indicam que a utilização do método PECS com indivíduos autistas foram eficazes. Dentro dos aspectos relatados tem-se o desenvolvimento da comunicação funcional, onde o indivíduo adquire o comportamento verbal não vocal, isto é, aprende a se comunicar funcionalmente. Há ainda, 
outros estudos que abordam o aumento do número de trocas de figuras de maneira independente, promovendo também a interação social, caráter de extrema importância para o indivíduo com autismo.

Logo, podemos perceber a exemplo disso no Quadro 1 e iniciando nossa discussão com Oliveira et al (2012) que os estudos dos quadros do transtorno do espectro do autismo apontam para uma grande variedade de alterações comportamentais em que a linguagem está profundamente relacionada às dinâmicas sociais e aos procedimentos repetitivos, refletindo em alterações cognitivas. As crianças apresentam severo prejuízo de compreensão de significado e no uso da linguagem verbal e não verbal. Assim, fica claro que a rápida aprendizagem das habilidades envolvidas no PECS ocorre devido ao contexto estruturado e concreto desse treino que facilita a compreensão da comunicação funcional pelos indivíduos com autismo.

Outra questão importante diz respeito ao sistema de comunicação alternativa que possibilitou à participante ampliar suas habilidades comunicativas e que vem mostrando no trabalho de Moreschi e Almeida (2012). Nele, as autoras relatam que com o início do processo de intervenção, e com a introdução do sistema de comunicação alternativa - PECS verificou-se que a participante passou a utilizar as figuras pictográficas como recurso comunicativo durante situações da vida diária. Embora a participante apresentasse comunicação verbal antes do início do processo de intervenção, essa comunicação não era eficiente durante as interações. Assim, a participante utilizou o sistema de comunicação como alternativa para aprimorar suas habilidades linguísticas, além de complementar sua comunicação oral. Logo, com base no relato das autoras, podemos inferir que a comunicação alternativa e suplementar também pode ser utilizada como suporte, para o desenvolvimento da linguagem e como recurso pedagógico em educação especial.

Todavia, os sistemas de comunicação se diferenciam quanto ao tipo de símbolos utilizados, se pictográficos, ideográficos ou arbitrários; ou ainda quanto ao número de símbolos que os compõem e sua forma de organização.

Com base nos estudos de Nunes e Santos (2015) que os dados coletados durante um atendimento pedagógico realizado em uma clínica, nos mostraram que no programa de intervenção a professora foi capacitada a usar o PECS associado a estratégias do ami para aumentar a frequência de interações com o aluno no decorrer das atividades pedagógicas e que um delineamento quase experimental registrou aumento na frequência de iniciativas de interação do aluno com a utilização dos pictogramas e com mudanças no estilo de interação da professora. Dessa forma, fica claro e como já mencionado anteriormente que o objetivo do PECS é ensinar indivíduos com déficit no repertório verbal a se comunicarem funcionalmente, isto é, a emitir comportamentos sob controle de estímulos antecedentes verbais ou não verbais e que produzam consequências mediadas por um ouvinte especialmente treinado para responder a estes comportamentos. Enfim, o menino aparentava ter boa compreensão de enunciados verbais, por isso o uso das figuras pareceu dispensável para auxiliar na compreensão de Pedro, que parecia assentir às instruções verbais da professora.

Além disso, outro estudo que merece destaque foi o de Cabral et al (2019) que nos mostraram sobre a questão do o uso do PECS como tecnologia do cuidado à criança com autismo. Com eles, podemos observar que a adoção do PECS frente a necessidade do desenvolvimento verbal infantil é imprescindível, ou seja, através do PECS, o indivíduo adquire o comportamento verbal não vocal, isto é, aprende a se comunicar funcionalmente emitindo respostas através de consequências mediadas por outra pessoa e por meio de figuras, fazendo a troca de imagens pelos objetos de interesse ou por algum outro reforçador generalizado. Além do mais, é importante salientar, segundo as autoras, a relevância do papel da família para que haja êxito na aplicação desse método com retorno satisfatório não só para o paciente, mas também para a sociedade como um todo.

Os resultados mostraram maior interação comunicativa do aluno na fase de intervenção, além de generalizar o uso do PECS-adaptado na sala de aula regular. Concluiu-se que a comunicação é um dos fatores fundamentais para que a inclusão escolar de um aluno com TEA ocorra de forma mais efetiva. Com tudo isso, o uso da comunicação alternativa e ampliada para estabelecer a comunicação de pessoas com TEA com ausência ou dificuldades na fala tem mostrado resultados positivos, com 
histórico de sucesso no seu uso, na medida em que as pesquisas vêm apresentando resultados positivos com significativa melhora no desenvolvimento da comunicação e da linguagem.

\section{Considerações finais}

Conclui-se que analisar a produção bibliográfica de trabalhos relacionados à aplicação do método PECS em pessoas com transtorno do espectro do autismo para saber quais os relatos descritos por meio da aplicação do método, nos foi bastante esclarecedora. A análise dos artigos obteve coerência nos resultados da utilização do método PECS com indivíduos autistas, sendo possível perceber ao se utilizar os sistemas de comunicação alternativa para a promoção da capacidade de comunicação do indivíduo, outras habilidades podem ser desenvolvidas, como habilidades acadêmicas, e com isso, as chances de inclusão social e educacional tendem a se expandir para essas pessoas que muitas vezes apenas não conseguem se fazer compreender utilizando a linguagem convencional de sua sociedade.

O treinamento para uso do programa é de suma importância, pois tem como objetivo ensinar comportamento verbal não vocal, para que crianças com déficits na comunicação possam requisitar coisas, produzindo consequências também no seu ambiente social. Isso se deve ao fato do surgimento das tecnologias que possibilitam a criação de recursos fundamentais que influenciam no melhor desenvolvimento cognitivo, pois possibilitam a comunicação entre pessoas com dificuldades de interação social. Tecnologicamente falando, os métodos mais utilizados com esse público, são os jogos educativos, utilizados não apenas como entretenimento, mas em diversas áreas com múltiplas finalidades, com o intuito de ajudar e facilitar o processo de ensinoaprendizagem de crianças com dificuldades.

Por outro lado, é significativo notar que todas as descrições de crianças autistas incluem alterações importantes na linguagem, especialmente no que se diz ao seu aspecto funcional. A questão da comunicação desses sujeitos representa provavelmente o seu distúrbio mais importante e que merece uma atenção especial para que sejam melhoradas essas dificuldades. Logo, assim, os sistemas de comunicação tornam-se alternativas na promoção de uma comunicação mais efetiva entre indivíduo sem comunicação oral e seu par, proporcionando meios funcionais de construção e partilha de ideias e sentimentos.

\section{Referências}

Cabral, A. B. S., Campos, A. C. S., Rodrigues, M. L. B., Bechara, S. L. F., \& Moraes, L. S. (2019). O uso do PECS como tecnologia do cuidado à criança com autismo. Revista Eletrônica Acervo Saúde, (31), e923.

Correa Netto, M. M. F. (2012). A comunicação alternativa - favorecendo a aprendizagem de criança com autismo, Aspeger e Angelman: formação continuada de profissionais de educação e saúde. 286f. Dissertação (Mestrado em Educação) - Faculdade de Educação, Universidade do Estado do Rio de Janeiro, Rio de Janeiro.

Daandels, N., Arboit, E. L., Van Der Sand, I. C. P. (2013). Produção de enfermagem sobre depressão pós-parto. CogitareEnferm, 18(4), 782-8. 20.

Minayo, M. C (2007). Pesquisa social: teoria, método e criatividade. (25a ed.): Vozes.

Mizael, T. M., Aiello, A. L. R. (2019). Revista eletrônica acervo saúde/ISSN 2178-2091. O uso do PECS como tecnologia do cuidado a criança com autismo. Google acadêmico.

Mizael, T. M., Aiello, A. L. R. (2019). Revisão de estudos sobre o PECS para o ensino de linguagem a indivíduos com autismo e outras dificuldades de fala Revista Brasileira. - pesquisa SciELO.

Moreschi, C. L., Almeida, M. A. (2012). A comunicação alternativa como procedimento de desenvolvimento de habilidades comunicativas. Rev. Bras. Ed. Esp., Marília, 18(4), 2012.

Nunes, D. R. P., Santos, L. B., Mizael, \& A. L. R. (2013). Aiello-revista brasileira de educação especial, Scielo Brasil. Revisão de estudos sobre o Picture Exchange communication system (PECS).

Nunes, D. R. P., \& Santos, L. B. (2015). Mesclando práticas em Comunicação Alternativa: caso de uma criança com autismo. Revista Quadrimestral da Associação Brasileira de Psicologia Escolar e Educacional, SP. 19(1).

Oliveira, G. C., Rosa, V. C. V., Carvalho, W., \& Freitas, E. F. (2015). Considerações da aplicação do método PECS em indivíduos com TEA. Estudos Goiânia, 42(3). 
Research, Society and Development, v. 10, n. 1, e33210111798, 2021 (CC BY 4.0) | ISSN 2525-3409 | DOI: http://dx.doi.org/10.33448/rsd-v10i1.11798

Rudio, F. V. (2001). Introdução ao projeto de pesquisa científica: Vozes.

Togashi, C. M., \& Walter, C. C. F. (2016). As Contribuições do Uso da Comunicação Alternativa no Processo de Inclusão Escolar de um Aluno com Transtorno do Espectro do Autismo. Rev. bras. educ. espec. [online].22(3), 351-366.

Vygotsky, L. S. (2001). A construção do pensamento e da linguagem: Martins Fontes.

Walter, C. C., \& Nunes, D. R. (2008). Estimulação da linguagem em crianças com autismo. In: Lamônica, DAC. (Org.). Estimulação de linguagem: aspectos teóricos e práticos: Pulso, 141-172. 